
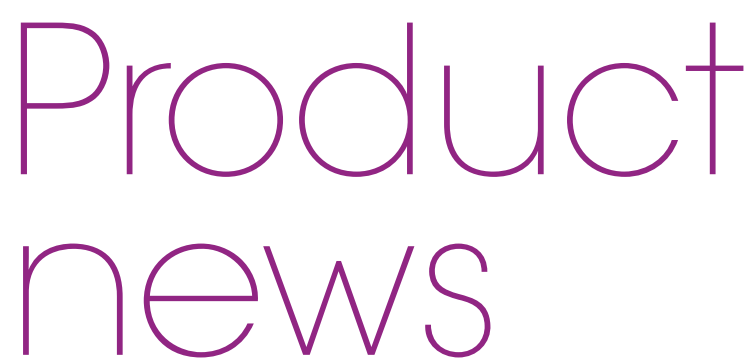

Product news is provided as a service to readers using text and images from the

manufacturer, supplier or distributor and does not imply endorsement by BDJ Team. Normal and prudent research should be exercised before purchase or use of any product mentioned.

\title{
BREAKTHROUGH TECHNOLOGY FOR A PROFESSIONAL CLEAN
}

On 26 June 2020, via a virtual launch event, Oral-B unveiled its most advanced rechargeable electric toothbrush yet: Oral-B iO.

Oral-B iO represents a step forward in creating the right balance between effectiveness and experience and reimagines how a brush performs, cleans and feels. From its revolutionary design to the harmony of its oscillation rotation and micro-vibrations technology, Oral-B iO wholly transforms brushing from a perceived 'chore' to a pleasurable experience that patients will love.

Oral-B iO's innovative key features include:

- Deep, but uniquely gentle clean: OralB's iconic round brush head contours each tooth, while the combination of oscillation rotations with the gentle energy of micro-vibrations allows Oral-B iO to glide tooth by tooth for a gentle clean, even along the gumline

- Pressure optimisation: The new Smart Pressure Sensor light provides positive reinforcement and protects gums by turning green when optimal pressure is applied and red when pressure is too hard

- Precision: The new innovative Frictionless Magnetic Drive system gently transfers the energy towards the bristle tips so that it is concentrated where it is needed most, to experience a deep, but uniquely gentle clean. The result, Oral-B's best ever clean with six times more effective plaque removal along the gumline [vs. a regular manual toothbrush].

Oral-B iO's Interactive Display is equipped with easy navigation through the brush features and allows users to personalise their brushing experience through a number of settings: welcoming greetings, choice of brushing modes, battery life icons, selection of preferred colour for the icons and Smart Ring, reminders to change the brush head and language settings. It further offers an enhanced, completely holistic brushing experience with a choice of brush colours

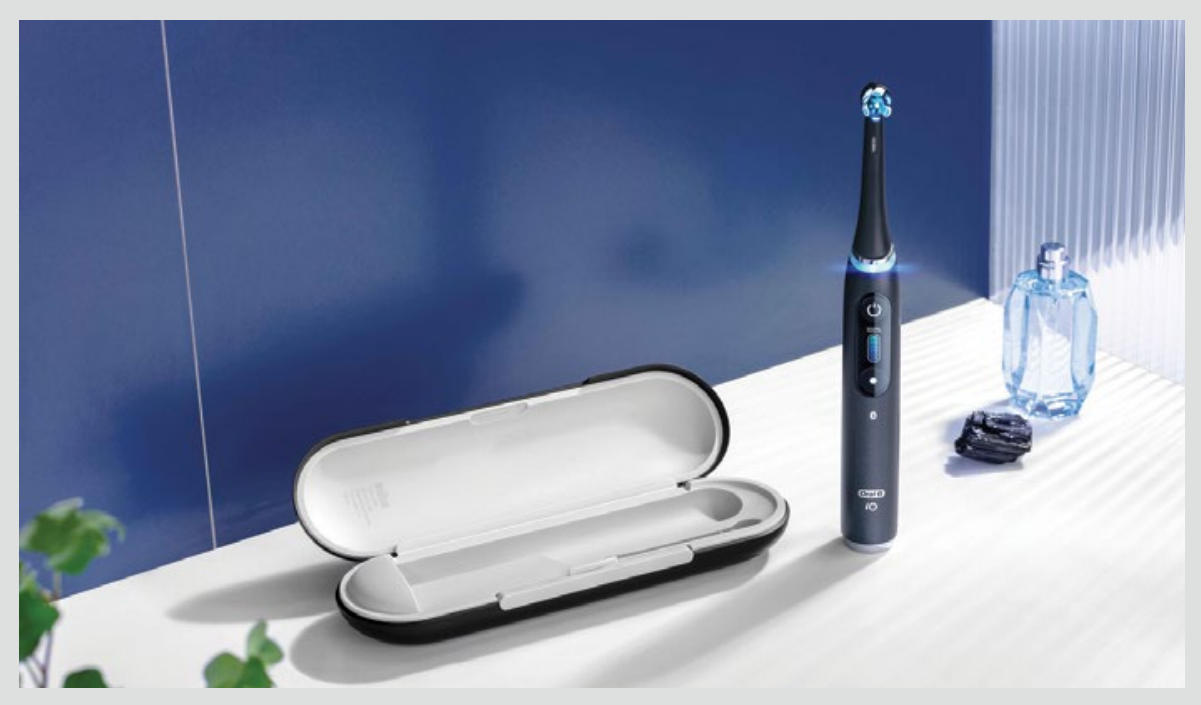

to fit the user's personality, a re-invented design and new, sleek magnetic charger, offering a full, long-lasting charge in about three hours.

Oral-B iO also comes with artificial intelligence, and is equipped with position sensors located in the handle. The Series 9 includes 3D teeth tracking technology with AI enabled brushing recognition to track the lingual and buccal areas of the three bottom and upper jaw areas, plus occlusal surfaces.

Oral-B iO's superior performance is evident in the results of an eight-week clinical study with peer reviews. In this study, and consistently with decades of clinical performance of Oral-B round head toothbrushes, Oral-B iO out-performed manual toothbrushes, providing a superior clean of teeth and gums. Compared to manual brushes, patients experienced:

- Twice as much plaque removal, and six times more plaque removal along the gumline

- Leading to improved gum health and $100 \%$ healthier gums in only one week.

The new Oral-B iO is a brush dental professionals can recommend with confidence, knowing their patients will get a truly one-of-a-kind brushing experience that will encourage them to brush longer and more effectively. In fact, in testing, patients using the Oral-B App, brushed an average of two minutes and 27 seconds per session compared to patients using a regular manual toothbrush, who averaged less than a minute.

For more information about Oral-B iO, visit https://contest.io.oralb.co.uk/ en-gb/?utm_source=OralB-Website\&utm_ medium $=$ Banner\&utm_campaign $=$ iOPrelaunch. 